\title{
Package models and the information crisis of prebiotic evolution
}

\author{
Daniel A. M. M. Silvestre, ${ }^{\text {a }}$ José F. Fontanari ${ }^{\text {a,* }}$ \\ ${ }^{a}$ Instituto de Física de São Carlos, Universidade de São Paulo, Caixa Postal 369, 13560-970 São Carlos SP, Brazil
}

\begin{abstract}
The coexistence between different types of templates has been the choice solution to the information crisis of prebiotic evolution, triggered by the finding that a single RNA-like template cannot carry enough information to code for any useful replicase. In principle, confining $d$ distinct templates of length $L$ in a package or protocell, whose survival depends on the coexistence of the templates it holds in, could resolve this crisis provided that $d$ is made sufficiently large. Here we review the prototypical package model of Niesert et al. (1981) which guarantees the greatest possible region of viability of the protocell population, and show that this model, and hence the entire package approach, does not resolve the information crisis. This is so because to secure survival the total information content of the protocell, $L d$, must tend to a constant value that depends only on the spontaneous error rate per nucleotide of the template replication mechanism. As a result, an increase of $d$ must be followed by a decrease of $L$ to ensure the protocell viability, so that the net information gain is null.
\end{abstract}

Key words: Prebiotic evolution, package models, hypercycles, error threshold, genetic diversity

\section{Introduction}

The information crisis in prebiotic or chemical evolution, as envisaged by Eigen (1971) in the 1970s, is grounded in two key observations: (i) the length of a replicating polymer (i.e., RNA-like template) is limited by the fidelity of replication (Eigen, 1971), and (ii) templates that differ from each other such that their differences reflect on their replication rates cannot coexist in a purely competitive setup (Swetina and Schuster, 1982). Despite some criticisms about the biological relevance of the information crisis (see, e.g.,Wiehe (1997); Wilke (2005)), the seminal work of Eigen has raised true questions about the onset of life, fueling the still unresolved debate on the mechanisms that explain how the primeval replicators coped with the loss of information resulting from competitive exclusion and recurrent mutations.

There are two competing scenarios that seek to solve the prebiotic information crisis by achieving template coexistence. In principle, template coexistence solves the information crisis because the total information content of the template pool is the product of the number of different templates and the maximum information coded per template, provided the template types have the same concentration. The first scenario is the hypercycle, a closed re-

\footnotetext{
* Corresponding author: fontanari@ifsc.usp.br
}

action scheme in which each replicating polymer aids in the replication of the next one, resembling a current motif in biochemical metabolic pathways (Eigen and Schuster, 1978). This scheme requires that the primordial replicators act both as templates and as cross-catalytic replicases, but this key assumption encounters strong criticism. In fact, since natural selection is responsive to replication rates only, one can expect it to make each element of the hypercycle a better target for the replicase (or a better replicase to itself), but one cannot expect natural selection to drive the cross-catalytic activity of the hypercycle components in the same way. Therefore, the likely fate of each replicase is to mutate into the so-called parasites - molecules similar to the modern genomic polymers which lack catalytic activity (Smith, 1979; Bresch et al., 1980). In addition, hypercycles with a large number of different templates are subject to large fluctuations in the concentrations of its members, rendering it susceptible to extinction by stochastic effects (Nuño and Tarazona, 1994; Campos et al., 2000). Nonetheless, the hypercycle model has received substantial empirical support regarding its basic premises as catalytic activity in a variety of biological molecules, including DNA and RNA, and the experimental synthesis of minimal self-replicating systems are now well established (see Paul and Jovce (2004)).

The alternative prebiotic scenario to address the template coexistence issue is the so-called package model 
approach, in which the templates are confined in packages termed protocells or prebiotic vesicles (Gabriel, 1960; Bresch et al., 1980; Niesert et al., 1981; Niesert, 1987; Silvestre and Fontanari, 2005; Fontanari et al., 2006). The basic assumption here is that a nonspecific replicase is codified and assembled by a number $d$ of distinct functional templates. Template replication is possible only with the aid of the replicase which, in turn, can be put together only when all template types are present in the package.

The combined dynamics of templates and protocells is in many aspects similar to the metapopulation dynamics used in the study of patches in ecology (see, e.g., Kevmer et al. (2000)). The distinguishing features of the package models are the random drift in the template dynamics due to the finite capacity of the protocells, and the assortment load resulting from the stochastic fission of the protocells. These very features that make the dynamics interesting, also make it much less amenable to analytic treatment. In fact, as compared with the hypercycle and quasispecies models for which the deterministic chemical kinetics equations suffice to describe most of the relevant phenomena (see, however, Alves and Fontanari (1998); Campos and Fontanari (1999)), package models like the classical model of Niesert et al. (1981) or the stochastic corrector model of Szathmáry and Demeter (1987) are not so well characterized and only recently slightly simplified versions of the original models were analyzed thoroughly (Grey et al., 1995; Zintzaras et al. , 2002; Silvestre and Fontanari, 2005; Fontanari et al., 2006; Silvestre and Fontanari, 2007).

Our main reservation about the package models premises concerns its group selectionist flavor. One way or the other, all aforementioned package models assume that the growth rate of the protocell lineage depends on the composition of the template population confined within each protocell. Although at first sight this may seem a reasonable assumption, it is not supported by experimental evidence: recent experiments with model protocells show that the growth of lipid vesicles does not depend on the nature of the substances they hold in (Hanczyc et al., 2003; Chen et al., 2004, 2006; Zaher and Unrau, 2007). A more plausible assumption is to admit that protocell fission is triggered when the total concentration of the templates reaches some critical value so that the integrity of the membrane is compromised (Chen et al., 2006).

Interestingly, the package model proposed in the pioneer work of Niesert et al. (1981) conforms to this more realistic scenario, as no connection is made between template composition and the mechanism of protocell fission. Alas, for purely technical reasons - to keep the protocell lineage to a size that could be manageable by their computational resources - those authors have introduced an extraneous ingredient into their model, namely, a prospective value which essentially gauges the survival probability of a protocell according to its template composition. This prospective value plays exactly the same role as the group selection pressure in models where a direct relation be- tween template composition and protocell fission (or protocell reproduction) is made explicit (Zintzaras et al. , 2002; Silvestre and Fontanari, 2005; Fontanari et al., 2006). To handle and quantify the unlimited growth of the protocell population we borrow a powerful tool from statistical physics, namely, the spreading or epidemic analysis used to characterize nonequilibrium phase transitions in lattice models (Grassberger and de La Torre, 1979). This technique suits particularly well to the task of locating the boundaries in the space of parameters that separate the subcritical regime, where the extinction of the lineage is certain, from the supercritical regime, where the probability of survival of the lineage is nonzero (see, e.g., Rosas and Fontanari (2003)).

Since ingredients such as prospective values for protocell survival or protocell fitness commonly used in the definition of package models are not supported by experimental evidence, we find the reexamination of the original version of the model of Niesert et al. (1981) absolutely essential. More importantly, because viable protocells (i.e., protocells that contain the $d$ functional template types) are allowed to reproduce unrestrainedly, the region of viability of the metapopulation is the greatest possible in this model. Any other package model that assumes protocell competition by assigning fitness values to the protocells according to their template compositions will exhibit a smaller region of viability.

We have found that the confinement of templates in packages does not solve the information crisis of prebiotic evolution because, to guarantee the viability of the protocells, the total information content of each package must remain constant, i.e., the product between the template lengths $L$ and the number of distinct templates $d$ is a constant that depends, essentially, only on the spontaneous error rate per nucleotide. Hence there is no information gain of increasing the number of coexisting templates types since their lengths must decrease proportionally.

The rest of the paper is organized as follows. In Sect. 2 we review the package model of Niesert et al. (1981) and in Sect. 3 we briefly describe the spreading analysis technique. The results are presented in a crescendo of difficulty from Sects. 4 to 6. In particular, Sect. 4 is dedicated to the study of the limit of perfect replication fidelity; Sect. 5]considers the effect of mutations to the so-called parasite class, which are essentially nonfunctional templates; and Sect. 6 the effect of mutations to the lethal class, which are precipitating agents that prevent the assemblage of a replicase. Finally, Sect. 7 is devoted to our concluding remarks.

\section{The package model of Niesert et al. (1981)}

According to the model of Niesert et al. (1981) we consider a metapopulation composed of a variable number of packages or protocells, each of which encloses a certain number of templates (RNA-like molecules) and a few polypeptides. Since all our simulations begin with a sin- 
gle mutant-free protocell we use the words metapopulation and protocell lineage interchangeably throughout this paper. There are $d$ distinct types of functional templates which, when present in the same protocell are capable of assembling a nonspecific replicase of finite processivity and finite fidelity of replication. As pointed out by Niesert et al. (1981), this kind of primitive translation is achieved by cooperation of RNAs with t-RNA character and an RNA with messenger function. Here we define the processivity $\Lambda$ of the replicase as the number of template copies it produces in some convenient unit of time, so $\Lambda$ can be viewed as a measure of replication efficiency as well. This definition can be made equivalent to the usual definition of processivity as the average number of nucleotides added by a replicase per association/disassociation with the template if $\Lambda$ is allowed to take on noninteger values and all templates have the same length. We recall that the replicase can only be formed in the presence of all $d$ types of template. If a protocell lacks a single functional template type it is considered unviable and discarded from the protocell population. In addition, all template types display identical targets to the replicase, which results in a neutral replication process, i.e., all template types have the same replication rate.

Since any plausible replication process is susceptible to errors, we must take into account the possibility of mutations. Two types of mutations are allowed in the model. First, there are mutations that produce non-functional templates which contribute nothing to the assembling of the replicase but keep their replication capability unharmed. These mutants are termed parasites. Second, there are lethal mutations which generate molecules that prevent the assembling of a replicase or, equivalently, produce a non-functional replicase. In any case, an offspring protocell that contains a mutant of this kind is rendered unviable. Mutations to the parasite type occur with probability $u$ whereas mutations to the lethal type occur with probability $v$. Reverse mutations or mutations between functional template types are not considered. The life cycle of the protocells consists of three events - template replication, protocell fission and protocell demise - which take place in this order and are described in the following.

\subsection{Template replication}

This process, which describes the replication of the templates inside the protocells, is regulated by one of the crucial parameters of the model, namely, the number of replicated molecules between two vesicle fissions $(\Lambda)$. As already pointed out, this quantity is the processivity of the replicase, i.e., the number of template copies the replicase can produce in a unit of time, taken here as the time between two consecutive fissions. The replication process is implemented as follows. For each protocell we choose a template at random and replicate it, returning both the original template and its copy to the protocell, and this procedure is repeated until the processivity of the replicase is reached, i.e., it is repeated exactly $\Lambda$ times. If the randomly selected template is a functional template then the copy will become a parasite with probability $u$ or a lethal with probability $v$. Hence the probability that the copy is perfect is $1-u-v$. If the selected template is a parasite, then the copy will become a lethal with probability $v$. Finally, in the case a lethal is selected, then the copy will also be a lethal. As a result of this procedure, exactly $\Lambda$ new templates are added to the protocell. We note that in the absence of mutations this process is exactly the Polya's urn scheme (Feller, 1968, Ch. V.2). The template replication procedure is repeated for all protocells in the metapopulation. In what follows we will refer to the number of templates inside a protocell as the size of the protocell.

\subsection{Protocell fission}

After template replication, the protocell is in excess of molecules since we assume that the membrane is impermeable to templates. As template replication is much faster than protocell fission, we envisage a scenario where fission begins at about the same time as the template replication cycle but terminates only after the replicase has exhausted its copying capacity. This means that the overall osmotic pressure tolerated by the protocell membrane is of the order of $\Lambda$, so that the fission process is triggered whenever the protocell size exceeds $\Lambda$. For example, if the size of a just generated daughter protocell is $n>\Lambda$ then the fission process starts but, by the time it is concluded, the protocell has already reached the size $n+\Lambda$.

Experimental models of lipid vesicles exhibit a variety of mechanisms for the fission process (Hanczyc and Szostak, 2004). Here we choose a mechanism that splits the mother protocell in two daughters and distributes templates of the mother vesicle between the two offspring at random. The random assortment of templates to the daughter vesicles may cause the loss of essential genes for survivorship (i.e., the assortment load), thus producing unviable protocells. To be more precise, if $S$ is the size of the mother protocell just before fission then the probability that the daughters have size $s$ and $S-s$ is given by the binomial $\left(\begin{array}{l}S \\ s\end{array}\right) 2^{-S}$.

\subsection{Protocell demise}

The viability of a daughter protocell is guaranteed provided that (i) it carries no lethal mutants, and (ii) it has at least one copy of each type of functional template. Any protocell lacking one of those templates or carrying a lethal mutant is dismissed because it is incapable of producing a working replicase.

\subsection{Metapopulation dynamics}

The sequence of the three processes stated above defines the time unit of the metapopulation dynamics. ¿From these processes it is clear that the size of the metapopulation 
(i.e. the number of protocells) can, in some cases, increase without bounds. In fact, the metapopulation dynamics can be viewed as a branching process with denumerably many types (Kimmel and Axelrod, 2002). As pointed out before, to circumvent this 'technical' difficulty which rapidly saturates the computer resources of the time, Niesert et al. (1981) have opted to discard supernumerary protocells according to an arbitrary prospective value which essentially gauges the odds of a protocell to leave viable descendents (see Sect. 55). In practice, using such a prospective value to eliminate the least promising protocells introduces an additional criterion for protocell demise, which can be interpreted as a selection pressure acting at the protocell level which favors protocells with high prospective values. Since the survival probability of the protocell begins to depend on specific details of their template compositions, the resulting model becomes very similar to the group selection models proposed to explain template coexistence (see, e.g., Fontanari et al. (2006)). Interestingly, Niesert et al. (1981) do not mention group selection or multi-level selection: although they state that the unit of selection is the entire package, they overlooked the conflict between package and template interests, especially the parasites. Here we do not use any prospective value to control the metapopulation size and the only criteria for elimination of a protocell is the presence of a lethal mutant or the lack of any type of functional template.

Although the number of protocells may grow unboundedly, the number of templates inside each protocell (i.e., the protocell size) fluctuates around a finite value, though, strictly, a viable protocell can assume any size value in the range $d, \ldots, \infty$. In fact, starting from whatever conditions the average number of templates per package will approach the processivity of the replicase very quickly. Consider, for example, the average number of templates $n_{t}$ in a protocell at time $t$. It is related to the average number of templates of its mother protocell $n_{t-1}$ by the recursion equation

$$
n_{t}=\frac{\left(n_{t-1}+\Lambda\right)}{2}
$$

whose solution is simply

$$
n_{t}=\frac{n_{0}-\Lambda}{2^{t}}+\Lambda,
$$

where $n_{0}$ is the size of the vesicle that originated the lineage. Hence, regardless of the ancestor size, after a few generations the average size of the protocells will equal the processivity value of the replicase.

The ultimate goal of the analysis of the metapopulation dynamics is to determine the regions in the space of the parameters $d$ (number of functional templates), $\Lambda$ (processivity of the replicase), $u$ (probability of mutation to parasites) and $v$ (probability of mutation to the lethal type) where the protocell lineage has a nonzero probability of thriving. To achieve that we resort solely to brute force simulations of the metapopulation dynamics as will be described in the forthcoming sections. A slightly modified version of this model, in which template replication takes place simultaneously through a Wright-Fisher process and the daughter protocells have fixed size $\Lambda$, yields to an analytical approach (Silvestre and Fontanari, 2007). In this contribution, however, we have opted to stick to the original model of Niesert et al. (1981) so as to complement and complete that classical work on the theory of prebiotic evolution.

\section{The spreading analysis}

The spreading or epidemic analysis put forward by Grassberger and de La Torre (1979) is the preferred technique of statistical physics to locate and characterize equilibrium as well as non-equilibrium phase transition lines. The method is tailored to population dynamics problems that exhibit an absorbing state (subcritical regime), where the protocell population has gone extinct, and an active state (supercritical regime) where the population undergoes exponential growth. Separating these two regimes there is the critical regime where the population growth (if any) is sub-exponential, usually a power law in the time variable.

The basic idea of the spreading analysis is to follow the evolution of the lineage that sprouts from a single mutantfree protocell of size $\Lambda$, in which the functional templates are evenly represented. For each time $t$ we carry out from $10^{6}$ to $10^{9}$ independent runs, all starting with the same ancestor protocell. The number of runs depends on the values of the control parameters, and is chosen so as to guarantee a representative number of surviving samples at any given time. Here we focus on the time dependence of two key quantities: the average number of protocells $N_{t}$ and the survival probability of the lineage $P_{t}$ calculated at time $t$. Clearly, $P_{t}$ is simply the fraction of runs for which there is at least one protocell in the lineage at time $t$. We note that in the calculation of $N_{t}$ we take an average over all runs, including those for wich the lineage has already gone extinct at time $t$.

Let $\Delta$ be a real variable that measures the distance to the critical point. For example, if the transition from the absorbing to the active phase takes place at the mutation probability $u=u_{c}$ when all other parameters are held fixed then $\Delta=u_{c}-u$. Close to this transition point, i.e., for $\Delta \approx$ 0 , and for sufficiently large $t$, we expect that the average number of protocells and the survival probability obey the scaling hypothesis

$$
\begin{aligned}
N_{t} & \sim t^{\eta} \phi_{N}\left(\Delta^{\nu} t\right) \\
P_{t} & \sim t^{-\delta} \phi_{P}\left(\Delta^{\nu} t\right)
\end{aligned}
$$

where $\eta, \delta$ and $\nu$ are non-negative critical dynamic exponents, and $\phi_{N}$ and $\phi_{P}$ are universal scaling functions, usually exponential functions (Grassberger and de La Torre, 1979). At the critical point $\Delta=0$, a log-log plot of $N_{t}$ (or $P_{t}$ ) as function of $t$ yields a straight line, the slope of which yields the dynamic exponent $\eta$ (or $\delta$ ). Upward and downward deviations from the critical straight line indicate supercritical and subcritical behaviors, respectively. The 
mere observation of these deviations allows a very precise estimate of the value the critical parameter $u_{c}$.

A particularly important quantity, as far as the characterization of branching processes is concerned, is the ultimate probability of survival of the lineage, defined as

$$
\Pi=\lim _{t \rightarrow \infty} P_{t}
$$

so that $\Pi=0$ in the critical and subcritical regimes $(u \geq$ $\left.u_{c}\right)$, whereas $\Pi>0$ in the supercritical regime $\left(u<u_{c}\right)$. In the latter case, for $u$ sufficiently close to $u_{c}$ we have another scaling relation, $\Pi \sim \Delta^{\beta}$ where $\beta$ is a static critical exponent. Since Eq. (4) holds for $t \rightarrow \infty$ in the sub as well as in the supercritical regime the critical exponents $\delta, \nu$ and $\beta$ must not be all independent. To see that we rewrite Eq. (4) in the supercritical regime as $P_{t} \sim \Delta^{\nu \delta} \psi_{P}\left(\Delta^{\nu} t\right)$ where $\psi_{P}(x)=x^{-\delta} \phi_{P}(x)$ is an arbitrary function that tends to a finite positive value when $x \rightarrow \infty$. It follows then that $\beta=\delta \nu$. The precise estimate of these exponents is a major concern of a branch of the statistical physical that deals with branching-like processes in lattice models (see, e.g., Marro and Dickman (1999)). The branching process we consider here can be viewed as a mean-field-like version of similar processes in lattice models and so the critical exponents are the mean-field ones: $\eta=0, \delta=-1$, and $\nu=\beta=1$. The a priori knowledge of these values can be useful to validate our estimate of the threshold locations, which are actually our main concern in this paper.

\section{The cost of diversity}

The information crisis of prebiotic evolution revealed by the quasispecies model is essentially a consequence of the limited fidelity of replication of the templates with the resulting steady accumulation of mutants in the population (the mutational load in the population genetics jargon). In package models there is an additional threshold phenomenon which, even in the absence of mutations, limits the amount of information stored in the molecular pool: the limited processivity of the replicase bounds the number of distinct template types that can coexist in a protocell. We refer to this new information crisis as the cost of diversity. Deterministic models, such as the quasispecies and the hypercycle models, assume that the processivity of the replicase is essentially infinite but a recent estimate of this parameter in artificially selected RNA polymerase ribozymes yields a processivity on the order of a few tens of nucleotides (Zaher and Unrau, 2007), which is insufficient to produce even the shortest functional ribozyme known, the hairpin ribozyme (Kun et al., 2005). To better characterize the processivity threshold in this section we focus in the case in which the replication fidelity is perfect, i.e., $u=v=0$.

Although some features of the primordial replicase such as its specificity and processivity are key elements in most prebiotic scenarios, their roles are rarely punctuated in the literature. Notable exceptions are Michod (1983) who has

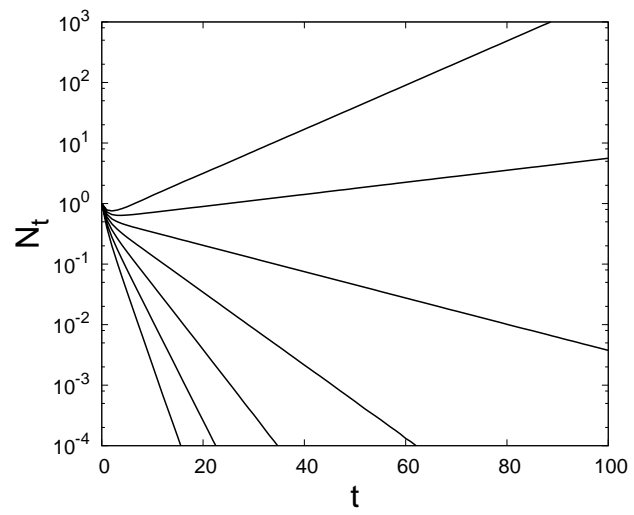

Fig. 1. Average number of protocells in a lineage originated in a single well-balanced and mutation-free founder. The parameters are $d=4$ and (from bottom to top) $\Lambda=4, \ldots, 10$. The data shown for each $t$ represents the average over $10^{7}$ independent runs. The semi-logarithmic scale facilitates the observation of the exponential increase and decrease of the lineage size in the sub and supercritical regimes.

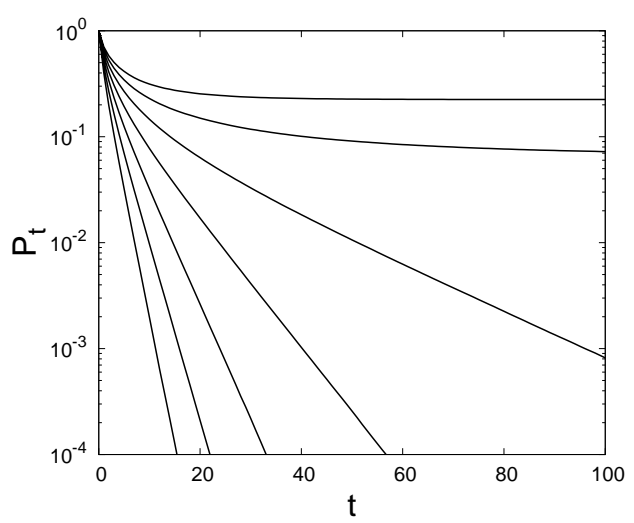

Fig. 2. Same as Fig. 1 but for the survival probability. For large $t$ this quantity tends to a constant value, the ultimate survival probability $\Pi$. The minimum value of the processivity $\Lambda$ that guarantees $\Pi>0$ is $\Lambda_{c}=9$ in this case.

argued that the lack of specificity of the primordial replicase gives the templates an altruistic-like character which could be maintained by the confinement of the templates in packages, and Niesert et al. (1981) who have, in turn, shown that a high processivity is necessary to make up for the assortment load and so to guarantee the coexistence of a few functional templates in the protocell.

To obtain the minimum value of the processivity $\Lambda$ of a replicase that needs $d$ functional templates for its assembling we use the spreading technique as illustrated in Figs. 1 and 2 for the case $d=4$. Given the discrete nature of $\Lambda$, the critical regime is absent in this case and so one observes a discontinuous jump from the typical subcritical behavior (both $N_{t}$ and $P_{t}$ vanish exponentially with increasing $t$ ) to the supercritical regime $\left(N_{t}\right.$ increases exponentially with increasing $t$ whereas $P_{t}$ approaches the ultimate survival probability $\Pi$ ). We recall that the calculation of $N_{t}$ includes the extinct runs as well and this is the reason that $N_{t}$ can take on values smaller than 1 in the subcritical regime (note that $N_{0}=1$ ). 


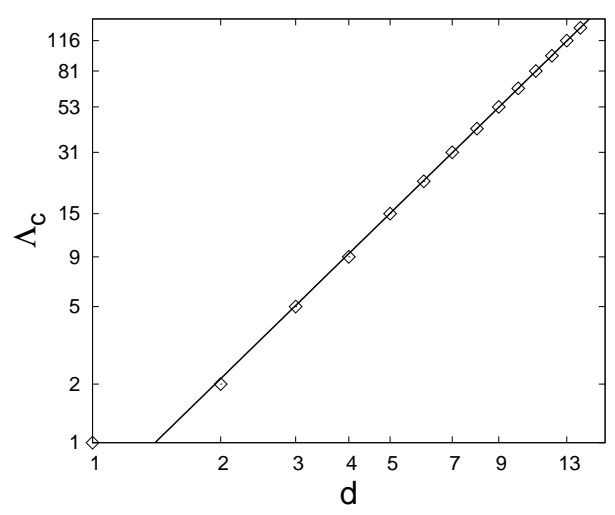

Fig. 3. Logarithmic plot of the minimum value of the processivity $\Lambda_{c}$ above which the protocell lineage has a finite probability of escaping extinction against the diversity $d$. The extinction of the lineage is certain for $\Lambda<\Lambda_{c}$. The solid line is the fitting $\Lambda_{c}=d^{2} / 2$. The parameters are $u=v=0$.

Repetition of the spreading analysis for different values of the diversity $d$ allows us to obtain the dependence of the minimum processivity $\Lambda_{c}$ with $d$. The results are summarized in Fig. 3, which essentially shows that for $d$ not too small the data is described very well by the fitting $\Lambda_{c}=$ $d^{2} / 2$. For $\Lambda<\Lambda_{c}$, the replicase is unable to produce sufficient copies of the templates to compensate for the stochastic loss of functional template due to the assortment load, and so the lineage goes extinct with probability one. For $\Lambda \geq \Lambda_{c}$, the lineage has a nonzero probability of ultimate survival.

As pointed out by Silvestre and Fontanari (2007), there is a simple combinatorial argument to explain the scaling $\Lambda_{c} \sim d^{2}$ at the critical boundary in the error-free replication case, that goes as follows. Assuming that the typical size of the protocells is $\Lambda$ then the number of different types of viable protocells is

$$
\left(\begin{array}{c}
\Lambda-1 \\
d-1
\end{array}\right)
$$

whereas the total number of protocell types is

$$
\left(\begin{array}{c}
\Lambda+d-1 \\
\Lambda
\end{array}\right)
$$

The logarithm of the ratio $r$ between these two quantities can be written as

$$
\ln r=\sum_{i=1}^{d-1} \ln \frac{1-i / \Lambda}{1+i / \Lambda} \approx-\frac{2}{\Lambda} \sum_{i=1}^{d-1} i \approx-\frac{d^{2}}{\Lambda},
$$

from where we can see that the only way to obtain nontrivial values of this ratio (i.e., $r \neq 0,1$ ) for large $\Lambda$ and $d$ is to assume that $d^{2} / \Lambda$ remains of order of 1 , which yields $r \sim \exp \left(-d^{2} / \Lambda\right)$. Clearly, $r \rightarrow 0$ corresponds the subcritical regime since the fraction of viable protocell types is vanishingly small in this case, whereas $r \rightarrow 1$ corresponds to the supercritical regime as practically all protocell types are viable.

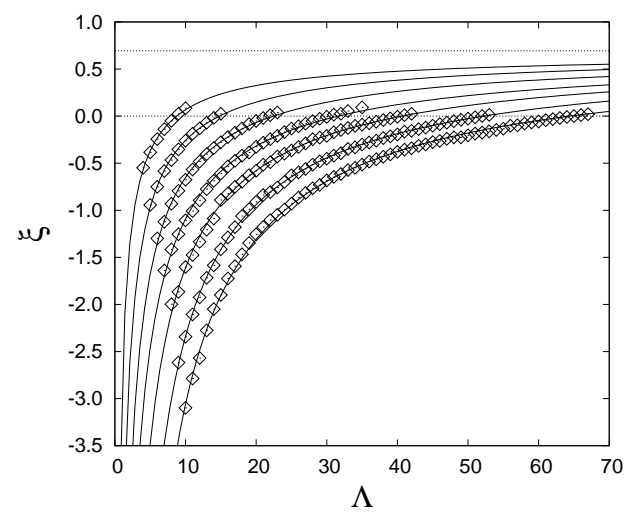

Fig. 4. Asymptotic growth rate of the lineage as function of the replicase processivity in the error-free case for (from top to bottom) $d=4, \ldots, 10$. The solid lines are the fittings $\xi=\ln 2+a \Lambda^{\theta}$ with $\theta<0$. The value of $\Lambda$ above which $\xi$ becomes positive defines the limits to the realm of viability of the lineage. The horizontal lines are $\xi=0$ and $\xi=\ln 2$.

The relation $\Lambda_{c} \sim d^{2} / 2$ is the same as that found in the original work of Niesert et al. (1981) as well as in the variant of Silvestre and Fontanari (2007), and it poses another challenge to the primordial protocells, for which no mechanism of segregation can be assumed to exist: the need to overcome the efficiency lower bound $\Lambda_{c}$ in order to maintain a given number of distinct functional templates. This threat to prebiotic evolution was aptly termed Charybdis by Niesert et al. (1981) in reference to the monster of the Greek mythology.

There is yet another difficulty that passed unnoticed in previous works and that makes the diversity cost transparent. From Fig. 1 we can easily see that $N_{t} \sim \exp (\xi t)$ for large $t$ where $\xi=\xi(\Lambda, d)$ is the asymptotic growth rate of the metapopulation, shown in Fig. 4. The point here is that for $\Lambda$ fixed, the growth rate decreases with increasing diversity, which means that there will be a selective pressure to increase $\Lambda$ while $d$ is kept to some minimum possible value. A way out of this conundrum would be to assume that the processivity of the replicase depends somehow on the information content of the template pool (i.e., $\Lambda=\Lambda(d)$ ) so that it would be impossible for a system of $d$ template types to assemble a replicase with processivity $\Lambda \gg \Lambda_{c} \sim d^{2} / 2$.

\section{The parasite load}

To better realize the implications of an imperfect replication mechanism, we will focus first on the mutations to the parasite type only, i.e., $u>0$ but $v=0$. The accumulation of parasites in a protocell does not make it unviable, but in the long run it can reduce the efficiency of the replicase, leading to the underproduction of functional template copies that could counterbalance the disruptive effect of the assortment load. (We recall that the parasites have the same replication rate as the functional templates).

Since the mutation probability $u$ varies continuously in the range $[0,1]$, in this section we can finally appreciate the 


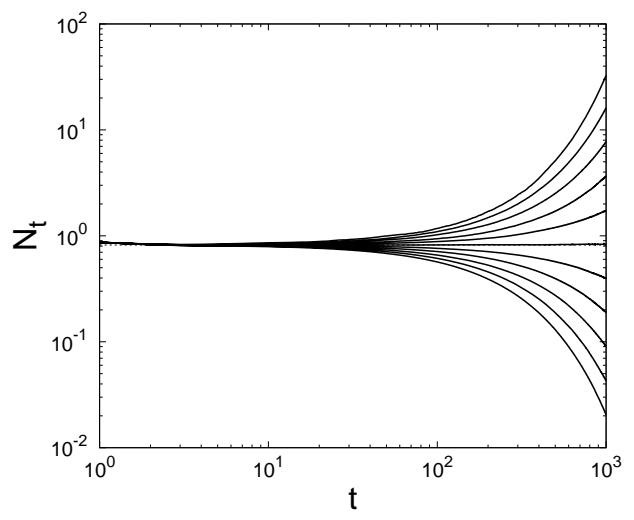

Fig. 5. Logarithmic plot of the average number of protocells in a lineage for several values of the parasite mutation probability (top to bottom) $u=0.180,0.181, \ldots, 0.190$. The critical mutation probability is $u_{c} \approx 0.185$ and corresponds to the horizontal straight line $(\eta=0)$. The parameters are $d=2, \Lambda=2$, and $v=0$.

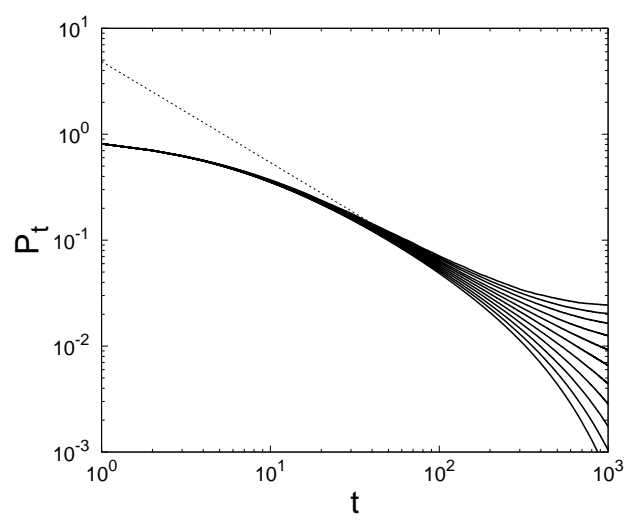

Fig. 6. Same as Fig. 5 but for the survival probability. The dashed straight line is the fitting $P_{t} \sim t^{-1}$ of the critical curve.

effectiveness of the spreading analysis to locate the critical mutation probability $u_{c}$ that separates the sub and supercritical regimes. Accordingly, in Figs. 5 and 6 we show log$\log$ plots of $N_{t}$ and $P_{t}$ for values of $u$ close to $u_{c}$. Visual inspection of Fig. [5 leads to the estimate $u_{c}=0.185 \pm 0.001$ for case $d=\Lambda=2$. As pointed out before, in the subcritical regime one has $N_{t} \sim \exp (\xi t)$ with $\xi \sim-|\Delta|^{\nu}$. The time decay constant $\xi$ is calculated by replotting Fig. 6 in the semi-log scale (see Fig. 2 for a similar graph) and then fitting the straight lines that result from this scale transformation. Finally, Fig. 7 confirms the linear dependence of $\xi$ on $|\Delta|$, and so the exponent value $\nu=1$. We conclude then that the ultimate survival probability vanishes as $\Pi \sim \Delta$ when the critical mutation probability is approached from the supercritical regime. These results assure us of the reliability and adequacy of the spreading technique to locate the mutation threshold in the package model of Niesert et al. (1981). This technical procedure was repeated for different values of the control parameters $d$ and $\Lambda$ in order to obtain a complete phase diagram of the model. Finally, we note that in this scheme we have imposed no limitation whatsoever to the total protocell population size.

Fig. 8 summarizes the dependence of the critical muta-

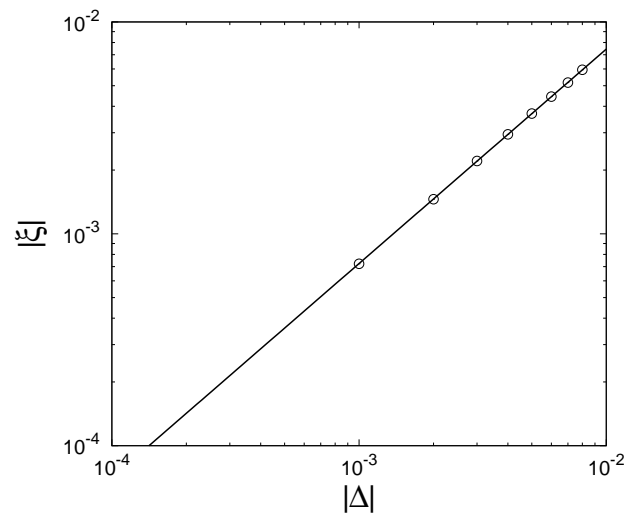

Fig. 7. Logarithmic plot of the absolute value of the time decay constant $\xi$ against $|\Delta|$ for the data shown Fig. 6 The slope of the straight line used to fit the data is $\nu=1.01 \pm .01$.

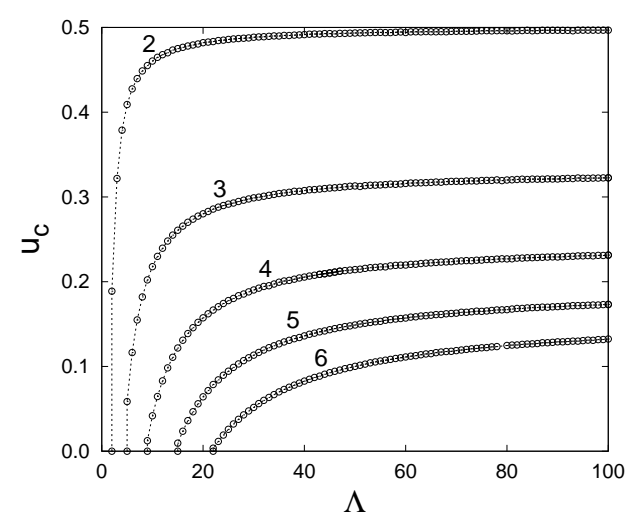

Fig. 8. Critical mutation probability to the parasite class $u_{c}$ as function of replicase processivity $\Lambda$ for values of the template diversity $d$ indicated in the figure. Mutations to the lethal class are no permitted $(v=0)$.

tion probability $u_{c}$ on $d$ and $\Lambda$. We recall that for $u \geq u_{c}$ the lineage is unviable, i.e., $\Pi=0$. For low diversity, the introduction of parasites has practically no effect on the viability of the lineage. Solely when the mutation probability takes very large values then the harm caused by the parasites becomes appreciable and must be compensated by the increase of the replicase processivity $\Lambda$. For high diversity, however, the fate of the lineage is much more sensitive to the presence of parasites, meaning that metapopulations with a high diversity of functional templates must have a very efficient replicase right at the beginning, which happens to be the situation of modern organisms. All polymerase cores involved in replication have similar processivity values, regardless of the number of genes per genome, which can vary from few hundreds genes in the smallest prokaryotes to tens of thousands in the largest eukaryotic genomes (Benkovic et al., 2001). We note, however, that the more sundry viral polymerases do not follow this scenario.

A salient feature of the metapopulation dynamics revealed by Fig. 8 is that, for fixed $d$, there is a value of the mutation probability beyond which coexistence is impossible regardless of the value of the replicase processivity. This is so because $u_{c}$ tends to the well-defined value $1 / d$ 


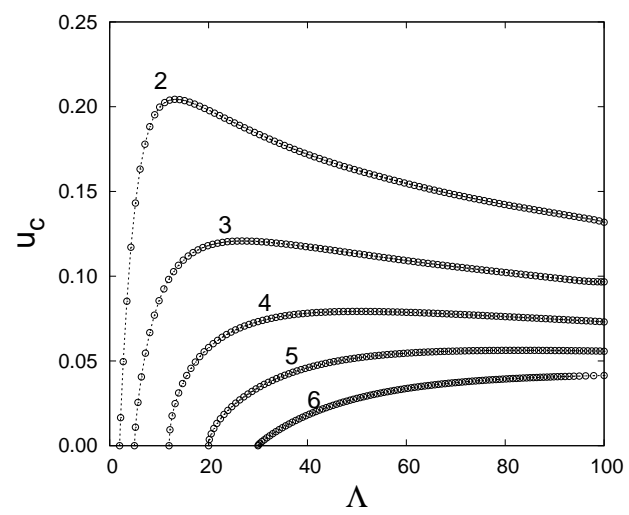

Fig. 9. Same as Fig. 8 but using the prospective value of Niesert et al. (1981) to discard supernumerary protocells. This additional ingredient leads to the incorrect prediction that high processivity values can be deleterious to the protocell population, in the sense of curtailing its region of viability.

in the limit $\Lambda \rightarrow \infty$. Nevertheless, contrary to the claim of Niesert et al. (1981), increasing $\Lambda$ is always benefic to the lineage: there are no 'mutational reefs' or Charybdis' partner - the monster Scylla - awaiting for the metapopulation. In fact, as pointed out before, their conclusion was a result of the prospective value used to limit the metapopulation size, which was based on three properties, namely, the degree of equipartition of the copies among the different functional templates, the number of parasites and the overall redundancy of the functional templates. This claim is confirmed by the result of the simulation of the metapopulation dynamics using the prospective value of Niesert et al. (1981) as shown in Fig. 9, from where we see that $u_{c}$ increases towards a maximum and then decreases towards zero as $\Lambda$ increases further. Rather surprisingly, the introduction of an explicit group selective pressure towards more balanced protocells turns out to be harmful for the metapopulation, which now can stand much lower values of the mutation probability. The reason is that for large $\Lambda$ and not too low $u$, the surviving vesicles in the supercritical regime are heavily loaded with parasites and so use of such selection criterion would purge them from the population resulting in the premature extinction of the lineage.

\section{The role of lethal mutants}

Lethal mutants are precipitating agents, the action of which disrupts the ability of the set of functional templates to assemble the replicase. Accordingly and following Niesert et al. (1981) we assume that the activity of the lethal mutants comes about only after the fission of the mother protocell, so that only the daughter protocells are affected by this type of mutant. This is a best case scenario, since there is a chance that one of the daughters comes out free of lethal mutants.

Since the mutation probability to the lethal class $v$ is a real variable, we can use the spreading technique to locate the critical value $v_{c}$ that separates the sub and the super-

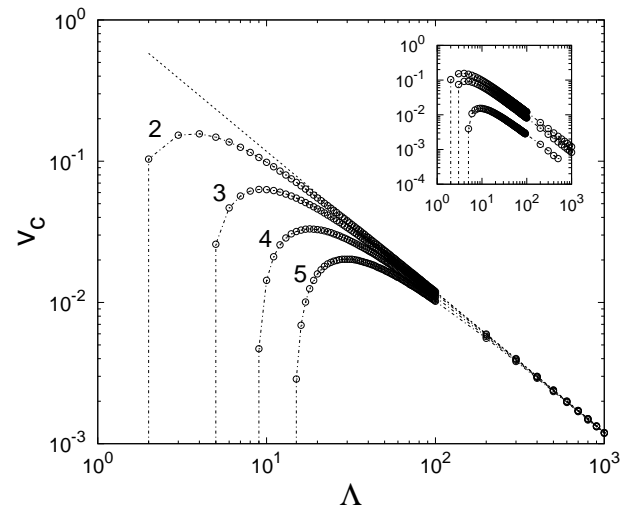

Fig. 10. Critical mutation probability to the lethal class as function of the replicase processivity in the absence of parasites $(u=0)$ for the values of the template diversity $d$ indicated in the figure. For large $\Lambda$ we find $v_{c} \sim 1 / \Lambda$ (dashed straight line) regardless of the value of $d$. The inset show $v_{c}$ vs. $\Lambda$ for $d=2$ and (top to bottom) $u=0,0.2$ and 0.4 , indicating that the asymptotic scaling $v_{c} \sim 1 / \Lambda$ is independent of $u$ as well.

critical regimes. The main graph of Fig. 10 summarizes our results in the case that mutations to the parasite class are forbidden $(u=0)$. In this case it is clear that too high processivity values are harmful to the metapopulation. A compromise between the 'fluctuation abyss' (loss of functional templates due to the assortment load) and the 'mutational reefs' (production of lethal mutants) is achieved by a finite value of $\Lambda$, which corresponds to the maximum in the curves of Fig. 10, It is interesting that $v_{c}$ becomes practically independent of $d$ in the limit of large $\Lambda$, where the data is very well fitted by the function $v_{c} \sim 1 / \Lambda$, which essentially means that the average number of lethal mutants must be smaller than 1 in any viable lineage. In fact, the relation $v_{c} \Lambda \approx 1$ holds in the case mutations to the parasite class are allowed as well, as shown in the inset graph of Fig. 10. The only effect of $u>0$ is to shift uniformly $v_{c}$ towards lower values. The important point illustrated by Fig. 10 is that for fixed $v$ the metapopulation is viable only between a certain range of processivity values, which excludes too small as well as too high values of $\Lambda$. This result is better illustrated in Fig. 11 which shows the dependence of $u_{c}$ on $\Lambda$ for $v$ and $d$ fixed.

We note that the presence of parasites does not add any qualitative new feature to the model dynamics: the threshold phenomenon for low $\Lambda$ is due to the assortment load only, whereas the threshold for high $\Lambda$ results solely from the destructive activity of the lethal mutants. This is in stark contrast with the major role played by $u$ in free-gene models, as evidenced by the error-threshold in the deterministic quasispecies model (Eigen, 1971) and Muller's ratchet in finite population models (Haigh, 1978). In fact, the analogue of the error threshold transition is the limiting value $u_{c} \sim 1 / d$ for large $\Lambda$ (see Fig. 8) which is irrelevant here because the presence of lethal mutants prevents the evolution of replicases with such high processivity values. Nonetheless, the existence of an error threshold-like transition between viable and unvi- 


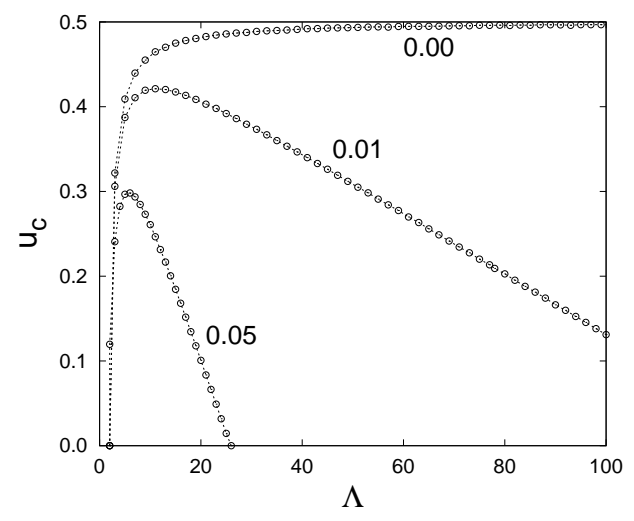

Fig. 11. Critical mutation probability to the parasite class as function of the replicase processivity for $d=2$ and $v$ as indicated in the figure. The upper bound on $\Lambda$ is due solely to the effect of lethal mutants, whereas the lower limit is due to the assortment load.

able states with respect to lethality contrasts with the absence of thresholds in the presence of lethal mutants in the quasispecies model (Wilke, 2005) and corroborates the findings of Takeuchi and Hogeweg (2007). The reason the steadily accumulation of parasite mutants does not lead to a phenomenon similar to the Muller's ratchet is that we have assumed a truncated fitness landscape at the protocell level, so protocell containing only parasites cannot accumulate. In any event, since the metapopulation can undergo unrestrained growth in the supercritical regime, the accumulation of parasites is not a serious hindrance as it is in the case the metapopulation size is fixed (see, e.g., Fontanari et al. (2003)).

\section{Conclusion}

The reexamination of the classic package model of Niesert et al. (1981) has revealed a few novel features and clarified some aspects of the metapopulation dynamics, which were obscured in the original work by the introduction of an artificial prospective value to limit the metapopulation size. To circumvent this difficulty, we have resorted to the powerful spreading technique of statistical mechanics which, by focusing on the time dependence of a few quantities, permits the precise location of the parameter values bordering the sub and supercritical regimes of population growth. In addition, our computational resources allow us to handle metapopulation sizes of order of $10^{6}$ packages, a figure that would be unthinkable in the beginning of the 1980s.

In the case of finite processivity $\Lambda$, which is the situation of interest in package models, we find that the parasites have no significant role in the evolution of the metapopulation and, in particular, mutations to the parasite class do not set an upper bond to the values that $\Lambda$ can assume. This is in disagreement with the findings of the original analysis (see Figs. 8 and 9). In fact, the phenomena responsible for the scenario 'life between Scylla and Charybdis' are the assortment load (i.e., the random assignment of templates to the daughter protocells) which sets a lower bound to $\Lambda$ and the presence of lethal mutants which, in turn, set the upper bound to $\Lambda$ (see Figs. 10 and 11).

An important result, which seems to have been completely overlooked by Niesert et al. (1981), regards the apparently unproblematic situation where the replication fidelity is perfect, i.e., $u=v=0$. The difficulty is illustrated in Fig. 4 which shows that the lower the diversity, the higher the growth rate of the metapopulation. So, in the case that two such metapopulations are set to compete in the same environment, it is evident that the lineage with higher diversity will inevitably be excluded. The situation becomes even worse in the presence of parasites and lethal mutants, since these mutants tend to cause more harm to the lineages with high diversity (see Figs. 8and10). This is a very unpleasant situation, the solution of which requires ad hoc assumptions about the protocell fitness (e.g., a fitness that increases with the template diversity) or about the dependence of $\Lambda$ on $d$ in order to revert the negative scenario revealed by Fig. 4

A word is in order about the amount of extra information eked out by confining the templates in packages. Here we equate information content with template length. The fundamental quantities here are the spontaneous error rate per nucleotide $\epsilon$, the molecule length $L \gg 1$, and the fraction of neutral single nucleotide substitutions $\lambda$ (see, e.g., Takeuchi et al. (2005)) from which we can readily obtain the probability that a template copy becomes nonfunctional (i.e., a parasite or a lethal),

$$
\mu=u+v=1-\exp [-\epsilon(1-\lambda) L] .
$$

A generous estimate of these parameters (see, e.g., Drake and Holland (1999); Johnston et al. (2001); Kun et al. $(2005))$ is $\epsilon \sim 0.005, L \sim 200$ and $\lambda \sim 0.25$ which yields $\mu \sim 0.53$. This is a truly disastrous result which precludes the coexistence of even two templates. Alternatively, we can use Eq. (9) to calculate the maximum length $L_{m}$ of a template in a best case scenario where lethal mutants are absent $v=0$ and the replicase processivity is infinite so that $u_{c} \approx 1 / d$. The result is $L_{m}=-[\ln (1-1 / d)] /[\epsilon(1-\lambda)]$, which, using the same parameter values as before, yields $L_{m} \sim 77$ for $d=4$, resulting thus in a total of about 300 nucleotides. Considering that this is a best case scenario, the improvement over the free-gene situation (about 200 nucleotides) is scanty. For instance, for large $d$ we have $L_{m} d \approx 1 /[\epsilon(1-\lambda)]$ which is essentially a constant value that depends on environmental factors only. Hence there is no information gain by increasing $d$ since the length $L_{m}$ of the coexisting templates must decrease in the same proportion so as to keep the total information content (i.e., $L d)$ constant. A similar information conservation principle' is likely to hold for the hypercycle model as well. We recall that in the case of the elementary hypercycle, the maximum number of templates that can coexist in a dynamical equilibrium state is $d=4$ (Eigen and Schuster, 1978). The analysis of the error propagation in the hypercycle (Campos et al., 2000) is not useful here because 
the error tail (mutant) class considered in that work is not equivalent to the parasite class of the package models, since those mutants do no receive catalytic support from the hypercycle members.

The original package model proposed by Niesert et al. (1981) and reviewed here is truly extraordinary for a simple reason, which perhaps passed unnoticed even to their proponents: because the protocells do not compete and the lineage is free to grow unrestrainedly, the region of viability of the metapopulation in this model is the greatest possible! Introducing the notion of protocell fitness or prospective survival value that depends explicitly on the template composition of the protocells, regardless of the prescription one chooses, can only reduce the size of that region. This is the reason that the critical mutation probability in Fig. 8 is greater than its counterpart in Fig. 9. This means that our estimates for the critical mutation probabilities $u_{c}$ and $v_{c}$ are upper bounds to the critical mutation probability of any package model for which the survival of the protocell is linked to the coexistence of a set of functional templates. Therefore, since even in this best case scenario the information gain derived from the coexistence of the distinct templates is not significant, this class of models should be discarded as possible solutions to the prebiotic information crisis. This is not to say that packages are not important in prebiotic evolution, since it is undisputable that compartments are essential to the evaluation of the translation products of the information coded in the templates (Eigen et al., 1980).

Coming up with a coherent scenario to explain the coexistence of distinct templates has proved to be a most difficult endeavor and it may already be time to turn to new approaches to solve the information crisis of prebiotic evolution. We have nothing to offer on this direction.

\section{Acknowledgements}

D.A.M.M.S. is supported by CAPES. The work of J.F.F was supported in part by CNPq and FAPESP, Project No. 04/06156-3.

\section{References}

Alves, D., Fontanari, J. F., 1998. Error threshold in finite populations. Phys. Rev. E 57, 7008-7013.

Benkovic, S. J., Valentine, A. M., Salinas, F., 2001. Replisome-mediated DNA replication. Ann. Rev. Biochem. 70, 181-208.

Bresch, C., Niesert, U., Harnasch, D., 1980. Hypercycles, parasites and packages. J. Theor. Biol. 85, 399-405.

Campos, P. R. A., Fontanari, J. F., 1999. Finite-size scaling of the error threshold transition in finite populations. J. Phys. A.-Mat. Gen. 32, L1-L7.
Campos, P. R. A., Fontanari, J. F., Stadler, P. F., 2000. Error propagation in the hypercycle. Phys. Rev. E 61, 2996-3002.

Chen, I. A., Roberts, R. W., Szostak, J. W., 2004. The emergence of competition between model protocells. Science 305, 1474-1476.

Chen, I. A., Salehi-Ashtiani, K., Szostak, J. W., 2005. RNA catalysis in model protocell vesicles. J. Am. Chem. Soc. 127, 13213-13219.

Chen, I. A., Hanczyc, M. M., Sazani, P. L., Szostak, J. W., 2006. Protocell: genetic polymers inside membrane vesicles, in: Gesteland, R. F., Cech, T. R., Atkins, J. F. (Eds.), The RNA World, Cold Spring Harbor Laboratory Press, New York, pp. 57-88.

Drake, J. W., Holland, J. J., 1999. Mutation rates among RNA viruses. Proc. Natl. Acad. Sci. U.S.A. 96, 1391013913.

Eigen, M., 1971. Selforganization of matter and evolution of biological macromolecules. Naturwissenschaften 58, 465523.

Eigen, M., Schüster, P., 1978. Hypercycle - principle of natural self-organization .c. realistic hypercycle. Naturwissenschaften 65, 341-369.

Eigen, M., Gardiner Jr., W. C., Schuster, P., 1980. Hypercycles and Compartments. J. Theor. Biol. 85, 407-411.

Feller, W., 1968. An Introduction to Probability Theory and Its Applications. Vol I. Wiley, New York.

Fontanari, J. F., Colato, A.,Howard, R. S., 2003. Mutation accumulation in growing asexual lineages. Phys. Rev. Lett. 91, 218101.

Fontanari, J. F., Santos, M., Szathmáry, E., 2006. Coexistence and error propagation in pre-biotic vesicle models: A group selection approach. J. Theor. Biol. 239, 247-256.

Gabriel, M. L., 1960. Primitive genetic mechanisms and the origin of chromosomes. Am. Nat. 94, 257-269.

Grassberger, P., de La Torre A., 1979. Reggeon field theory (Schlgl's first model) on a lattice: Monte Carlo calculations of critical behavior. Ann. Phys. (N.Y.) 122, 373396.

Grey, D., Hutson, V., Szathmáry, E., 1995. A reexamination of the stochastic corrector model. P. Roy. Soc. B - Biol. Sci. 262, 29-35.

Haigh, J., 1978. The accumulation of deleterious genes in a population - Mullers ratchet. Theoret. Population Biol. 14, 251-267.

Hanczyc, M. M., Fujikawa, S. M., Szostak, J. W., 2003. Experimental models of primitive cellular compartments: Encapsulation, growth, and division. Science 302, 618622.

Hanczyc, M. M., Szostak, J. W., 2004. Replicating vesicles as models of primitive cell growth and division. Curr. Opin. Chem. Biol. 8, 660-664.

Johnston, W. K., Unrau, P. J., Lawrence, M. S., Glasner, M. E., Bartel, D. P., 2001. RNA-catalyzed RNA polymerization: Accurate and general RNA-templated primer extension. Science 292, 1319-1325.

Keymer, J. E., Marquet, P. A., Velasco-Hernandez, J. X., 
Levin, S. A., 2000. Extinction thresholds and metapopu-

$167-181$. lation persistence in dynamic landscapes. Am. Nat. 156, 478-494.

Kimmel, M., Axelrod, D. E., 2002. Branching Processes in Biology. Springer, Berlin.

Kun, A., Santos, M., Szathmáry, E., 2005. Real ribozymes suggest a relaxed error threshold. Nat. Genet. 37, 10081011.

Marro, J., Dickman, R., 1999. Nonequilibrium Phase Transitions in Lattice Models. Cambridge University Press, Cambridge (UK).

Michod, M. R., 1983. Population Biology of the First Replicators: On the Origin of the Genotype, Phenotype and Organism. Amer. Zool. 23, 5-14.

Niesert, U., 1987. How many genes to start with - a computer simulation about the origin of life. Origins Life Evol. Biosph. 17, 155-169.

Niesert, U., Harnasch, D., Bresch, C., 1981. Origin of life between Scylla and Charybdis. J. Mol. Evol. 17, 348-353.

Nuño, J. C., Tarazona, P., 1994. Lifetimes of small catalytic networks. Bulletin of Mathematical Biology 56, 875-898.

Paul, N., Joyce, G. F., 2004. Minimal self-replicating systems. Curr. Opin. Chem. Biol. 8, 634-639.

Rosas A., Fontanari, J. F., 2003. Spatial dynamics and the evolution of enzyme production. Origins Life Evol. Biosph. 33, 357-374.

Silvestre, D. A. M. M., Fontanari, J. F., 2007. Preservation of information in a prebiotic package model. Phys. Rev. E 75, 051909.

Silvestre, D. G. M., Fontanari, J. F., 2005. Template coexistence in prebiotic vesicle models. Eur. Phys. J. B 47, 423-429.

Smith, J. M., 1979. Hypercycles and the origin of life. Nature 280, 445-446.

Swetina, J., Schuster, P., 1982. Self-replication with errors: A model for polynucleotide replication. Biophys. Chem. $16,329-345$.

Szathmáry, E., Demeter, L., 1987. Group selection of early replicators and the origin of life. J. Theor. Biol. 128, 463486.

Takeuchi, N., Hogeweg, P., 2007. Error-threshold exists in fitness landscapes with lethal mutants. BMC Evol. Biol. 7.

Takeuchi, N., Poorthuis, P. H., Hogeweg, P., 2005. Phenotypic error threshold; additivity and epistasis in RNA evolution. BMC Evol. Biol. 5, 9.

Wiehe, T., 1997. Model dependency of error thresholds: the role of fitness functions and contrasts between the finite and infinite sites models. Genet. Res. 69, 127-136.

Wilke, C. O., 2005. Quasispecies theory in the context of population genetics. BMC Evol. Biol. 5, 44.

Zaher, H. S., Unrau, P. J., 2007. Selection of an improved RNA polymerase ribozyme with superior extension and fidelity. RNA 13, 1017-1026.

Zintzaras, E., Santos, M., Szathmáry, E., 2002. 'Living' under the challenge of information decay: the stochastic corrector model vs. hypercycles. J. Theor. Biol. 217, 